\title{
La normalización de la calidad alimentaria y su regulación ${ }^{1}$
}

\section{Victor Manteca Valdelande \\ Doctor en Derecho}

En el presente trabajo se examina la regulación normativa del concepto de calidad alimentaria en la normas ISO partiendo del concepto genérico de lo que, actualmente se entiende por calidad en la producción y en la industria agroalimentaria, seguido de una análisis del control de riesgos y puntos críticos así como la de los planos normativos en materia de calidad agroalimentaria (internacional, europeo y nacional) para concluir con el análisis de un proyecto de establecimiento sobre gestión de la calidad.

\section{1) LA GESTIÓN DE LA CALIDAD EN LA ACTUALIDAD}

Las organizaciones empresariales de carácter industrial o comercial ofrecen productos y servicios al objeto de satisfacer las necesidades de los clientes cuyas expectativas se han hecho más concretas y diversificadas debido al incremento de la competitividad en el mercado y la diversificación de la oferta. Para situarse a la cabeza de los competidores y mantener buenos resultados económicos, es preciso utilizar sistemas de gestión de la calidad que produzcan mejoras continuadas, incrementado la satisfacción de los clientes de la organización y otras partes interesadas.

El sistema de gestión de una empresa está influido por sus objetivos, por sus productos y por prácticas específicas de dicha organización por ello los sistema de gestión de calidad varían de una organización a otra.

Uno de los grandes propósitos de la gestión de calidad consiste en mejorar los sistemas y procesos de manera que puedan conseguirse mejoras continuas en la calidad de los productos.

\footnotetext{
${ }^{1}$ El presente trabajo es una versión reducida de la memoria presentada por el autor en las Jornadas sobre evaluación de gestión de la calidad, Madrid febrero 2005. Configuración del sistema regulador de la calidad agroalimentaria en España.
} 
Al igual que otros sistemas de gestión, el sistema de gestión de calidad persigue la consecución de resultados en relación con los objetivos de calidad, incluyendo necesidades, expectativas y requisitos exigidos por las partes interesadas.

La empresa u organización tiene numerosos objetivos tales como el crecimiento, financiación, beneficios, mejora del entorno y seguridad en el trabajo; como los objetivos de calidad constituyen un complemento de estas metas, los sistemas de gestión de calidad pueden ser parte integrante del sistema de gestión empresarial.

El empleo de un sistema común se traduce en una simplificación de los planes, una asignación más sencilla de los recursos, una definición de objetivos comunes y una evaluación relevante de la eficacia general de la organización. Por ello puede llevarse a cabo una revisión del sistema basada no en meros requisitos legales sino en los que exija el propio sistema de gestión de la empresa. Además, si se lleva a cabo con referencia a un sistema certificado en el que deban cumplirse los requisitos del cliente y además los legales, deberá ser auditado conforme a los requisitos de las normas ISO 9001 o 40001 complementados por los de la normas 19011. Estas auditorías pueden llevarse a cabo como auditorías combinadas o conjuntas si procede.

\section{2) EL CONCEPTO DE CALIDAD EN LA INDUSTRIA AGROALI- MENTARIA}

Los procedimientos de control sanitario de alimentos están evolucionando hacia un sistema de calidad total donde el éxito requiere tener control sobre el proceso, materias primas, medio ambiente y formación de personal en los procesos de producción. Este sistema preventivo permite la fabricación de productos alimenticios con un alto grado de garantía de inocuidad.

Un sistema de calidad total de la empresa agroalimentaria debiera constar de los siguientes componentes:

- Las buenas prácticas de fabricación y distribución (BPFD) junto con el sistema de análisis de riesgos y control de puntos críticos (ARCPC), conformarían la gestión de calidad de alimentos en la empresa (sistemas que son obligatorios en la Unión Europea y Estados Unidos).

- Esta gestión alimentaria formaría parte de la gestión de calidad global, donde cabría el sistema ISO 9000 de carácter voluntario. 
Este sistema ARCPC garantiza la calidad del producto por medio del control de los procesos productivos, que son el núcleo de la ISO 9001 por ello la aplicación del sistema ARCPC es perfectamente compatible y debe estar integrado dentro de los sistemas de control de calidad como es la serie de ISO 9000 .

El ARCPC garantiza la calidad del producto por medio del sistema de la ISO 9000. Las normas ISO abarcan la gestión global de las firmas pero no ofrecen especificaciones técnicas para productos. Por ello es preciso recurrir a una fuente alternativa de conocimientos especializados y a la normativa correspondiente de aplicación. El sistema ARCPC proporciona este conocimiento especializado y la base para establecer un sistema de control.

Estos sistemas ARCPC (calidad ISO 9000 y calidad total) están relacionados con las denominadas Buenas Prácticas de Fabricación BPF y Buenas Prácticas de Distribución (BPD) que constituyen un requisito previo para poder llevar a efecto la aplicación del sistema ARCPC.

\section{3) EL MODELO EUROPEO DE CALIDAD}

El modelo europeo e calidad total es un sistema voluntario impulsado por la Fundación Europea de gestión de la calidad (EFQM) y la Comisión Europea. El modelo aprobado consta de nueve elementos que representan los criterios utilizados para evaluar el progreso de una organización hacia el estado de excelencia los elementos se agrupan en dos grupos: Agentes (criterios de 1 a 5) y resultados (criterios de 6 a 9). El modelo, en su versión más moderna, subraya la importancia de la innovación y el aprendizaje. Entre los agentes se encuentra el personal, la política y estrategia empresarial, las colaboraciones y recursos dando lugar a una serie de procesos; los resultados por su parte se clasifican en resultados de personal, resultados de clientes y resultados de sociedad. En definitiva los agentes significan lo que la organización hace y los resultados lo que la organización consigue, existiendo entre ambos planos un íntimo nivel de relación.

El modelo no supone una contradicción con los anteriores sistemas de gestión de calidad (ISO y ARCPC) sino como una síntesis o integración de ellos en un parámetro global de calidad total. 
De todos modos los sistemas de gestión de la calidad no deberían considerarse de forma aislada sino pasar a formar parte de una estrategia para mejora del funcionamiento de las empresas, junto con la calidad se ha propugnado la consideración de otros asuntos como el medio ambiente y la seguridad laboral.

Si la empresa u organización adopta un modelo de excelencia, como por ejemplo el premio europeo a la calidad, la diferencia entre ese sistema y el sistema basado en la norma ISO 9001 es que el primero incluye criterios de comparación mediante evaluación de los resultados lo cual es válido para todas las actividades y las partes afectadas de la organización. De todos modos ambos tipos de modelo permiten identificar los puntos fuertes y débiles de la organización, contienen requisitos genéricos de evaluación, proporcionan base para mejoras permanentes y conllevan un reconocimiento externo.

\section{4) NORMATIVA ISO EN MATERIA ALIMENTARIA}

La Organización Internacional de Normalización (International Standars Organisation ISO) es una federación internacional de organismos nacionales de normalización que ha desarrollado un conjunto de normas sobre normalización de cumplimiento voluntario para el establecimiento de sistemas de gestión de calidad en diversos sectores económicos.

La familia de normas ISO 9000 utilizada en todos los países miembros de este sistema primero se aprueba como norma internacional y después se desarrolla en cada país miembro.

Las normas e informes técnicos cubren todas las áreas de importancia relativas al sistema de calidad.

El comité técnico 176 de la Organización Internacional de Normalización elabora normas de gestión de la calidad por medio de la actividad de grupos de trabajo y subcomités cuyos miembros son representantes de comités nacionales donde se proponen nuevos elementos de trabajo o borradores de normas que son examinados por el Comité técnico y enviados a los miembros ISO para su ratificación como Borrador de Norma Internacional adoptándose posteriormente como norma nacional por los miembros ISO.

La empresa que adopta un sistema de gestión de calidad en la distribución de responsabilidades, de participación de los empleados en las metas de 
la organización, de regularidad en la organización de tareas y en la calidad del producto, de una mayor eficacia y eficiencia interna consigue una mejor imagen en el mercado lo cual contribuye a reforzar la confianza de la clientela y el beneficio empresarial.

Las normas ISO 9000 no son una imposición administrativa sino una exigencia del mercado, lo cual quiere decir que a pesar de su carácter voluntario, las grandes empresas alimentarias se dirigen solo a proveedores que cumplen estos requisitos por lo cual en la práctica se convierten en normas necesarias para poder acceder al mercado en buenas condiciones de aceptación.

Entre las ventajas que ofrece la titularidad de una certificación ISO podemos citar las siguientes:

- Una ampliación de la credibilidad de los clientes o de mercados extranjeros que ven en la certificación un modo de homologación plausible y ello redunda en una ampliación de las oportunidades de negocio.

- La adaptación que exige el sistema ISO puede suponer una disminución de costes en función de la clase de materiales empleados y del ajuste de procedimientos de fabricación.

- Ofrece una mayor posibilidad de control de riesgos de producción.

\section{5) LAS NORMAS ISO 9000}

El repertorio de normas ISO 9000 puede asegurar la calidad del producto y aumentar la rentabilidad de la empresa agroalimentaria pero no garantiza, totalmente, el control completo de los productos y su inocuidad salvo que su aplicación se combine con otro tipo de medidas complementarias como buenas prácticas de fabricación y distribución (BPF y BPD) y un sistema de análisis y riesgos y control de puntos críticos (ARCPC).

Las normas ISO 9000 dirigidas al establecimiento de sistemas de gestión de calidad son las siguientes:

- Norma ISO 9000:2000 sobre fundamentos y vocabulario de los sistemas de gestión de la calidad, contiene los fundamentos de los sistemas de gestión de la calidad especificando la terminología para dichos sistemas. Ha sustituido a las normas ISO 8402:1995 y 9000:1:1994. Describe los conceptos genéricos de los sistemas de gestión 
de calidad especificando la terminología como complemento se han publicado las Directrices para la selección y el uso en forma de publicación oficial ISO.

- Norma ISO 9001:2000 sobre requisitos de los sistemas de gestión de la calidad, constituye un modelo en cuanto a requisitos que deben satisfacerse para lograr un certificado o llevar a cabo un determinado contrato. Sustituyó a las normas ISO 9001, 9002 y 9003:1994.

- Norma ISO 9004:2000 sobre directrices para la mejora del desempeño de los sistema de gestión de la calidad sustituyó a las normas ISO 9004-1:1994,9004:1994-2,9004-3:1993,9004-4:1993 y 90044/CORR:1994.

- Norma ISO 19001:2002 que establece directrices de aplicación en las auditorías de sistemas de gestión de la calidad y/o medioambiental

Ya hemos indicado que estas normas ISO tienen un ámbito de aplicación internacional y existe una versión oficial en el ámbito español de cada una de ellas: norma UNE-EN ISO 9000 aprobada por el Comité Europeo de Normalización (CEN) del que forman parte los organismos de normalización de los Estados miembros.

\section{6) NORMA 9000:2000 PRINCIPIOS DE GESTIÓN DE CALIDAD}

La norma ISO 9000:2000 contiene ocho principios de gestión de la calidad que constituyen el cuerpo de doctrina de este sistema, cuyo éxito exige que la organización sea gestionada de forma sistemática y bien organizada.

1) El cliente como objetivo de la organización empresarial de modo que la satisfacción de las necesidades y la superación de las expectativas de aquel es el objetivo final de la empresa.

2) Necesidad de liderazgo a fin de establecer la unidad de acción y dirigir la organización que involucre a todo el personal en los objetivos.

3) Participación del personal en los objetivos, de modo que los empleados de todas las categorías sean considerados como la esencia de la organización. Por ello la implicación contribuye al total aprovechamiento de sus capacidades en beneficio de la empresa.

4) Gestión de recursos y actividades como un proceso para alcanzar los objetivos. Un resultado deseado podrá conseguirse de forma más eficaz si los recursos y las actividades asociadas al mismo se gestionan como un proceso coherente. 
5) Aplicación de los criterios de gestión. Identificar, comprender y gestionar un sistema de procesos interrelacionados con objeto de alcanzar un objetivo determinado para contribuir a la eficacia y eficiencia del sistema.

6) Proceso continuado de mejora de la calidad.

7) Fundamentación de la toma de decisiones en la información y análisis de datos.

8) Relación con los proveedores para implicarlos en los objetivos de calidad.

Además el apartado 2 de la norma ISO 9000:2000 regula los aspectos genéricos relativos a los sistemas de gestión de la calidad:

- Base racional para los sistemas de gestión de la calidad.

- Requisitos para los sistemas de gestión de la calidad y requisitos para los productos.

- Enfoque de los sistemas de gestión de la calidad.

- Enfoque basado en procesos.

- Política y objetivos de calidad.

- Papel de la alta dirección en los sistemas de gestión de calidad.

- Documentación.

- Evaluación de los sistemas de gestión de calidad.

- Mejora continuada.

- Papel de las técnicas estadísticas.

- Sistemas de gestión de la calidad y otros sistemas de gestión.

- Relación entre los sistemas de gestión de la calidad y los modelos de excelencia.

Además las normas ISO distinguen entre requisitos que deben cumplir los sistemas de gestión de la calidad y los requisitos que deben cumplir los productos. Los primeros se especifican en la norma ISO 9001 tienen carácter genérico y son susceptibles de aplicación a organizaciones de cualquier sector económico o industrial. Esta norma no establece requisitos para los productos que pueden ser establecidos por los clientes, por la propia empresa agroalimentaria o por condiciones reglamentarias.

Los requisitos del sistema de gestión de calidad contenidos en esta norma se refieren al propio sistema de gestión de la calidad, a la responsabilidad de la dirección, a la gestión de los recursos, a la realización del producto y/o servicio y al procedimiento de análisis medición y mejora. 
La versión aprobada en 2000 introdujo una nueva estructura manteniendo los requisitos del texto anterior: integra las veinte reglas existentes introduciendo nuevos requisitos basados en el concepto de mejora continua y satisfacción del cliente subrayando especialmente el papel coordinador de la dirección.

\section{6) LAS NORMAS ISO 9001:2000 REQUISITOS DE LOS SISTEMAS DE GESTIÓN DE LA CALIDAD Y 9004:2000 DIRECTRICES PARA LA MEJORA DEL DESEMPENO DE LOS SISTEMAS DE GES- TIÓN DE LA CALIDAD}

Esta norma especifica los requisitos de un sistema de gestión de la calidad en la que la empresa debe demostrar la capacidad organizativa para proporcionar de forma constante productos que satisfagan los requisitos reglamentarios y las exigencias del cliente incluyendo procesos de mejora y prevención de la no conformidad.

Además la norma ISO 9001:2000 se aproxima a la norma 14001:1996 sobre especificaciones y directrices para utilización de los sistemas de gestión medioambiental lo cual incrementa la compatibilidad de ambas normas en beneficio de los usuarios.

Las normas ISO 9001:2000 y 9004:2000 pueden aplicarse juntas o por separado y aunque no tienen el mismo ámbito, si participan de la misma estructura, la primera especifica requisitos de un sistema de gestión de la calidad que la empresa puede implantar internamente, tanto como requisito para poder establecer un contrato de suministro u otro como base para la certificación del producto. La norma ISO 9004, por su parte, proporciona una orientación sobre un espectro más amplio de objetivos en un sistema de gestión de la calidad con objeto de obtener una mejora continuada de la organización. Es decir se trata de una mera guía para implantar la norma anterior.

La estructura de estas normas es la siguiente:

- Introducción.

- Objeto y campo de aplicación. La norma especifica los requisitos de un sistema de gestión de calidad siempre que una empresa necesite demostrar su capacidad para proporcionar productos y servicios que satisfagan requisitos legales y de la clientela y aplique un proceso de automejora y de prevención de los productos o servicios no conformes. 
- $\quad$ Normas para consulta: Normas ISO 9000 y 9001.

- Términos y definiciones.

- Sistema de gestión de calidad. La empresa debe describir, implantar, mantener y mejorar, continuadamente, el sistema de gestión de calidad. La implantación incluye identificación de procesos necesarios al sistema, determinación de los criterios d ejecución y gestión eficaz y las medidas de seguimiento y análisis. Además la documentación debe incluir los objetivos de calidad de la empresa en forma escrita, un manual de calidad (que describa el alcance del sistema, los procedimientos documentados y una descripción de la interacción entre procesos del sistema), documentos precisos sobre los diferentes procesos y los requisitos de calidad que la norma requiera. En cualquier caso, debe establecerse un procedimiento documentado relativo a los registros de calidad que incluya la identificación, almacenamiento, protección, periodo de conservación y eliminación.

- Responsabilidad de la dirección. Que supone un compromiso previo con el desarrollo y mejora del sistema de gestión de la calidad, garantizando que la política de calidad es adecuada para los objetivos de la empresa, incluye la mejora continuada y la satisfacción de requisitos, traza el marco para el establecimiento y revisión de los objetivos de calidad está asimilada por la organización y es revisada periódicamente.

- Gestión de los recursos (humanos, infraestructuras y ambiente laboral).

- Realización del producto, que se articula en las siguientes fases: a) planificación de los procesos necesarios para elaborar el producto en función de los objetivos de calidad, b) establecimiento de procesos de producción, c) directrices en materia de diseño y desarrollo: establecimiento, resultados, revisión, validación y control, d) disposiciones sobre compras: proceso de adquisición garantizada y verificación, e) Elaboración de productos y prestación de servicios que incluye la fase de control incluyendo información sobre las características del producto, uso de equipos adecuados, instrucciones de trabajo, implantación del sistema de seguimiento y medida y de actividades de liberación, entrega y posteriores a la entrega; validación de procesos de producción que demuestren la capacidad de conseguir los objetivos planificados; Identificación y trazabilidad ( siempre que sea procedente la empresa identificará adecuadamente el producto a lo largo de toda su realización, identificando el estado del mismo con respecto a los requisitos de seguimiento y medida, controlando y registrando la identificación única del producto en los casos en que 
la trazabilidad sea un requisito. Además la empresa debe establecer los dispositivos de seguimiento y medida necesarios para proporcionar evidencias sobre la conformidad del producto con determinados requisitos.

- Medición análisis y mejora (de los procesos productivos, de la percepción del cliente, mediante auditorías internas). El control del producto no conforme se lleva a cabo mediante la definición de procedimientos documentados que establezcan controles, responsabilidades y autoridades relacionadas con el tratamiento de productos no conformes.

\section{7) LA NORMA ISO 9004:2000}

Esta norma establece que el propósito de una empresa u organización en materia de calidad consiste en:

- Identificar y satisfacer las necesidades y expectativas de la clientela y de otras partes afectadas por la producción (empleados, proveedores, propietarios y la misma empresa).

- Alcanzar, mantener y mejorar el rendimiento general de la organización y sus capacidades.

Una vez delimitado el objeto regulador y el ámbito de aplicación, la norma contiene una serie de definiciones que fijan con seguridad el significado de diferentes conceptos:

- Proceso es el sistema de actividades que utilizan recursos para transformar entradas en salidas.

- Producto es el resultado de un proceso.

- Gestión de calidad es el sistema para establecer los objetivos y el modo de su consecución.

- Planificación de la calidad es la parte dirigida a fijar objetivos y especificar los recursos y procesos necesarios para obtener los objetivos.

Entre los requisitos generales que debe cumplir un sistema de gestión de calidad se encuentran la identificación y diferenciación de los procesos de fabricación, así como su secuencia e interacción, el establecimiento de criterios y métodos precisos para que la ejecución y el control de procesos sean eficaces, la disponibilidad de recursos e información precisa para el funcionamiento del sistema, el seguimiento, medición y análisis de procedimientos y las acciones para la mejora continuada del sistema. 
La organización que establezca el sistema debe documentar los diferentes procedimientos mediante un Manual de calidad y un sistema de registros sometido a control.

El apartado dedicado a responsabilidad de la dirección define los compromisos de la dirección empresarial comprometida en el control de calidad y la forma de llevarlos a efecto además la dirección debe identificar, definir y comprender las necesidades y derechos del cliente incluyendo por supuesto los requisitos legales.

La política de calidad que se establezca en la empresa debe estar adecuada al propósito de la organización y los objetivos que se propongan deben tener presente el concepto de mejora continuada siendo actualizados regularmente.

La Dirección debe establecer unos objetivos medibles y coherentes con la política general de calidad adoptada y con la planificación aprobada. Además debe definir las responsabilidades de la organización y establecer los medios de participación del personal en la política de calidad de la empresa.

El sistema será revisado periódicamente teniendo en cuenta los resultados de auditoria, las peticiones de la clientela, introduciendo medidas correctoras y preventivas de mejora.

El establecimiento de un sistema de gestión de calidad requiere, además, el aporte e identificación de una serie de recursos materiales, humanos y funcionales. Los primeros deben definirse comunicando al personal sus funciones y responsabilidades determinando las necesidades de formación.

Respecto a los segundos la infraestructura es el más relevante y debe cumplir unos requisitos mínimos en función de las exigencias del sistema de gestión de calidad.

Entre los recursos funcionales deben identificarse los métodos y condiciones en que el trabajo debe desarrollarse en la empresa y además debe implantarse un sistema de gestión del conocimiento.

Respecto al capítulo de la elaboración del producto requiere una previa descripción de la secuencia de procesos de fabricación. Además deben identificarse los requisitos exigidos por los clientes incluyendo las obligaciones legales relativas al producto, los procedimientos de solución de controversias y el sistema de relaciones e información con los clientes. 
Los proveedores deben ser previamente evaluados por la empresa de manera que las materias primas adquiridas cumplen los requisitos exigidos por el sistema de gestión de calidad.

También debe haber un control de la producción validando los procesos de elaboración e identificación e implantado un sistema de trazabilidad cuya aplicación, por otra parte, se ha convertido en una obligación general de Derecho alimentario del de la entrada en vigor el Reglamento CE 178/2002 (artículo 18). Cuando se haya especificado en los objetivos deberán establecerse normas para almacenamiento, conservación manipulación y embalaje del producto.

Todo esto requiere además que, periódicamente, se lleven a cabo operaciones de supervisión de los equipos de medición prueba y control. Un proceso de medición efectivo debe demostrar la eficacia del sistema de gestión de la calidad, también debe llevarse acabo un seguimiento del grado de satisfacción del cliente con el producto y sistema.

Los datos de control deben ser analizados de cara a la mejora continuada de la eficacia del sistema.

\section{8) NORMA ISO 19011:2002 DIRECTRICES PARA LA AUDITORIA DE SISTEMAS DE GESTIÓN DE LA CALIDAD Y/O MEDIOAM- BIENTAL}

Se trata de una norma que constituye una verdadera guía para realización de auditorías y formación de auditores en el ámbito de calidad y presenta la siguiente estructura:

- Introducción que subraya la auditoria como herramienta de la dirección para efectuar un seguimiento de la implantación de la política de gestión de la calidad o medioambiental en una empresa u organización. Si bien se trata de una norma dirigida preferentemente a la realización de auditorías en el campo de la calidad y el medio ambiente, también es susceptible de aplicación a otro tipo de auditorías como la auditoría de procesos y la de productos.

- Objeto y campo de aplicación, que se extiende los fundamentos de la auditoría en este ámbito, la programación y la calificación de los auditores de gestión de calidad y medioambiente. 
- Referencia normativas, especialmente las normas ISO 9000:2001 y 14050:1998.

- Términos y definiciones: son validos los contenidos en las dos normas mencionadas y además se incluyen 14 definiciones adicionales.

- Fundamentos de auditoría. Incluyéndose los siguientes principios: Conducta ética, presentación imparcial de resultados, cuidado profesional debido, independencia y pruebas de conclusiones.

- Gestión de un programa de auditoria, que comprende apartados como los objetivos y alcance de programa, las responsabilidades, recursos y procedimientos, la implantación del programa, los registros y su seguimiento y revisión.

- Actividades de auditoria: inicio, revisión inicial de documentos, actividades in situ, informe de auditoria, finalización y seguimiento.

- Calificación de los auditores de sistemas de gestión de calidad y medioambiental, incluyendo conocimientos y habilidades exigidos, atributos personales, educación, experiencia laboral, formación y experiencia auditora, mantenimiento y mejora de conocimientos y proceso de evaluación de auditores.

La directriz incluye tres tablas sobre educación, formación y experiencia laboral y auditora recomendadas, métodos de evaluación y ejemplos de métodos para evaluar la competencia de los auditores.

El cumplimiento de las normas ISO 9000 por parte de una empresa adherida es verificado por los organismos de certificación reconocidos por el sistema ISO internacional que por lo general dependen de los organismos nacionales de normalización en España la Asociación española de normalización y certificación (AENOR) entidades de carácter independiente y privado de las cuales hemos hablado en otra ocasión en estas páginas.

\section{9) EL SISTEMA DE ANÁLISIS DE RIESGOS Y CONTROL DE PUN- TOS CRÍTICOS (ARCPC)}

El sistema ARCPC es un sistema para asegurar la inocuidad de los alimentos que tienen base científica y enfoque preventivo y es utilizado para la identificación, evaluación y control de los peligros encontrados durante las fa- 
ses de producción, procesado, manufacturas, almacén y uso de alimentos, se trata de un mecanismo que complementa los sistemas de calidad implantados a través de las normas ISO 9000 si bien se trata de una norma que no es susceptible de ser certificada. Este sistema surgió hace dos décadas en el ámbito de investigación seguido por la NASA para garantizar las condiciones sanitarias de los alimentos que debían ser ingeridos por los astronautas. Se trata de un sistema que pretende asegurar la sanidad de un alimento, desde el campo, la granja o el buque de pesca hasta la mesa del consumidor final. Como el recorrido es muy variado, considera que, desde el punto de vista de la calidad, hay puntos en los que no existe peligro alguno, otros en los que hay alto riesgo de que se produzcan contaminaciones que pueden afectar a la salud de los consumidores de alimentos; detectar y controlar los lugares y segmentos de la cadena en que pueden producir o difundir, las contaminaciones y en consecuencia evitarlas es el fin primordial perseguido por este procedimiento.

Se trata de un sistema de control de calidad que permite adelantarse a los riesgos propios de los procesos de producción, dando normas concretas y sencillas que deben seguir los trabajadores que intervienen en la elaboración para evitar peligros, además este sistema supuso una superación de los anteriores sistemas de inspección mediante muestreos aleatorios del producto final considerados no preventivos.

Este sistema ha sido adoptado por la Federal Drug Administration de los Estados Unidos y por la Comisión Europea, además ha sido objeto de análisis en el seno de la FAO, de la Organización Mundial de la Salud; además los principios del sistema que fueron aprobados, hace diez años, por el Codex Alimentarius fueron los siguientes:

- Análisis de los riesgos alimentarios potenciales de todas las operaciones afectadas en el marco de las desarrolladas por cada empresa.

- Localización en el espacio y en el tiempo de los puntos del proceso en los que puedan producirse riesgos identificados.

- Necesidad de elaborar un diagrama de flujo del proceso, señalando puntos en los que pueden aparecer los peligros y establecer medidas preventivas.

- Determinar entre los puntos de riesgo, los que son decisivos para garantizar la seguridad de los alimentos (Puntos críticos de control).

- Definir y aplicar procedimientos eficaces de control y seguimiento de los puntos críticos. 
Por ello es preciso establecer una serie de límites admitidos para cada punto crítico de control, fijando los criterios de vigilancia y frecuencia de controles, designar un responsable de su cumplimiento, determinar acciones correctoras cuando se detecte una desviación que supere el límite establecido y determinar el destino de los productos fabricados mientras el proceso se hallaba fuera de control.

La Directiva 91/493/CEE sobre normas aplicables a la producción y a la puesta en el mercado de los productos pesqueros fue el primer texto normativo comunitario que incluyó este sistema y dos años más tarde, con la Directiva 93/43/CEE relativa a las guías prácticas correctas de higiene de los productos alimenticios se estableció de forma general con carácter obligatorio. Esta Directiva se transpuso al Derecho español mediante el Real Decreto $2207 / 1995$ por el que se establecen normas de higiene relativas a los productos alimenticios y en el que se determina que en los controles que lleven a cabo las autoridades competentes para comprobar que se está cumpliendo con el sistema, se tomarán como referencia las guías prácticas correctas ya elaboradas y evaluadas favorablemente. Además las inspecciones oficiales de las Administraciones públicas deberán atender, especialmente, a los puntos críticos determinados por las empresas.

El sistema ARCPC fue específicamente diseñado para las industrias alimentarias y afines y se basa en un enfoque sistemático de la higiene del producto para prevenir y reducir los riesgos a lo largo del proceso productivo, tiene el mismo fundamento y estructura que las normas ISO: establecimiento de un plan, ejecución y control e introducción de medidas correctivas (de reacción o prevención). El espíritu de este sistema se basa en la anticipación de los riesgos asociados con la producción o empleo de los alimentos y la identificación de los puntos en los que pueden controlarse los riesgos; en esencia se trata de la racionalización de los controles precisos para asegurar un nivel adecuado de higiene alimentaria, anticipándose a los riesgos e identificando los puntos en que dichos riesgos puedan existir por eso ha resultado una alternativa, mucho más efectiva, a los sistemas clásicos de control.

Supone una evolución de los sistemas reactivos a los sistemas de prevención.

Hasta hace poco el sistema de control de los alimentos se ha basado, exclusivamente, en la inspección de las instalaciones y análisis de los productos finales por los servicios oficiales de la Administración pública. Este sistema ha resultado insuficiente y ha evolucionado hacia procedimientos de control so- 
bre el proceso productivo basado en el análisis de riesgos que aparecen en determinadas actividades industriales y evitar su aparición.

En este nuevo enfoque los servicios responsables del control alimentario deben verificar que las empresas apliquen el sistema ARCPC cuya aplicación garantiza un levado grado de seguridad y calidad.

El sistema es aplicable a todos los eslabones de la cadena alimentaria: producción, procesado, transporte y comercialización hasta el uso final en establecimientos y en el hogar.

Fases que forman el sistema ARCPC:

- Análisis de riesgos: Identificación de riesgos asociados a la producción alimentaria en todas las fases desde la producción, procesado, elaboración, distribución y consumo; valoración de su importancia y probabilidad de aparición. Los factores más importantes que deben considerarse en esta fase son las fuentes potenciales de contaminación, los perfiles de temperatura y el ph del producto, además de otros entre los que podemos mencionar el diseño higiénico del equipo, los procesos de limpieza y desinfección y estado sanitario del personal.

- Determinación de los puntos críticos de control (PCC) en los que los riesgos pueden ser controlados. Un PCC es una medida, procedimiento o práctica susceptible de ser controlada hay dos tipos de PCC uno que asegura el control de un riesgo o peligro y otro que reduce al mínimo el riesgo aunque no lo controla totalmente.

Cuando un riesgo pueda ser controlado en varios puntos debe decidirse cual será el punto más efectivo, además la clase y número de PCC son muy variables en función del tipo de industria y de los productos elaborados, no obstante, con carácter general, los puntos críticos de control en una industria son seis:

1) Recepción de materias primas teniendo en cuenta su posible contaminación por pesticidas, fertilizantes, etc.

2) Formulación de ciertos componentes del producto: ph, sales, etc.

3) Operaciones de procesado, principalmente aquellas que tengan efecto sobre las propiedades físicas o químicas del alimento: enfriamiento, calentamiento, secado, acidificación, concentrado, etc.

4) Empaquetado y almacenamiento. 
5) Higiene de los manipuladores.

6) Limpieza y desinfección de utensilios e instalaciones.

- Especificación de los criterios que indican que una operación se encuentra bajo control de un determinado PCC. Estos criterios pueden ser necesidades de tiempo y temperatura, ph, acidez, nivel de cloro, temperatura durante el proceso e distribución y almacenamiento.

- Establecimiento y aplicación de procedimientos para comprobar que cada PCC funciona correctamente. Se usan cinco tipos de procedimientos: Observación visual, valoración sensorial, determinaciones físicas, análisis químico y examen microbiológico. Las observaciones pueden incluir desde la del propio producto hasta las instalaciones y su estado de higiene.

- $\quad$ Aplicación de medidas correctoras en caso necesario.

- Acciones de verificación o supervisión del control y buen funcionamiento.

Estas fases se incluyen en los manuales de aplicación del sistema de análisis de riesgos y control de puntos críticos elaborados con la colaboración de la administración y diversas organizaciones empresariales del sector agroalimentario: productos cárnicos, industrias lácteas, conserva vegetales, bollería rellana, helados, cervezas, productos pesqueros congelados, platos preparados, bebidas envasadas, azúcar, aceitunas de mesa, vegetales congelados, zumos de frutas, aceites comestibles, etc.

Los manuales, por su parte, constituyen una guía de carácter general para todas las industrias que deben adaptarse a las características específicas exigidas para cada caso.

\section{0) GUÍAS DE BUENAS PRÁCTICAS}

El cumplimiento de los sistemas ARCPC es apoyado por Guías de buenas prácticas de higiene y fabricación (BPF) que facilitan el establecimiento de procedimientos que controlan los requisitos de operación en un establecimiento ofreciendo condiciones ambientales que favorecen la producción de alimentos inocuos. Las Guías de buenas prácticas constituyen un requisito previo a la aplicación del sistema ARCPC.

El Código de regulaciones federales de la Federal Drug Administration norteamericana contiene un repertorio de buenas prácticas clasificadas en cuatro 
apartados de los cuales el primero define las cuestiones más importantes incluyendo el punto crítico de control y describiendo las prácticas de conducta e higiene personal. En el segundo, se encuentran incluidos los requisitos para el mantenimiento del espacio y condiciones de construcción, así como las condiciones de ventilación e iluminación, el control de plagas, uso y almacenamiento de productos químicos, incluyendo desinfectantes, suministro de agua, distribución de tuberías y tratamiento de residuos. El tercer apartado expone las condiciones generales de los equipos incluyendo los requisitos de construcción, mantenimiento y limpieza. El cuarto apartado contiene directrices sobre controles en la producción siendo la parte más detallada del código toda vez que regula el proceso debe manejarse de modo higiénico tomando precauciones adecuadas para prevenir la contaminación del producto. Se establecen controles en cada fase de producción.

\section{1) MARCO NORMATIVO SOBRE CALIDAD E INOCUIDAD ALI- MENTARIA}

El marco normativo que afecta a la empresa agroalimentaria está compuesto por tres escalones: el internacional, el comunitario europeo y el español en sus aspectos de normativa estatal, autonómica y local.

\section{1- a) Normativa internacional}

En este plano las principales normas que deben tenerse en cuenta en el ámbito de la calidad alimentaria son el Codex alimentarius y los convenios multilaterales en materia alimentaria. El primero es el resultado efectivo de la comisión del Codex alimentarius (compilación de normas alimentarias de carácter voluntario, códigos de buenas prácticas y directrices) creada en el seno de la Organización de Naciones Unidas para la Agricultura y la Alimentación (FAO) y la Organización Mundial de la Salud (OMS) hace cuatro décadas. Con un número de Estados miembros superior a los 160 el Codex ha pasado a ser considerado como una de las instituciones alimentarias de mayor influencia en el ámbito internacional. Pues cada vez es mayor el número de países que adapta su Derecho alimentario nacional a los criterios establecidos por el Codex (especialmente en lo que se refiere a los aspectos relativos a la inocuidad).

La Comisión del Codex elaboró el Código General de prácticas y principios de higiene de los alimentos o Código de Buenas prácticas de manufactura (BPM) donde se recomienda la aplicación del sistema de análisis de riesgos 
y control de puntos críticos (ARCPC) con el propósito de elevar el nivel inocuidad alimentaria.

Posteriormente a la creación del Codex y con la puesta en marcha de la Organización Mundial de Comercio (OMC) fueron aprobados dos convenios internacionales de relevancia en materia de calidad alimentaria: el Acuerdo sobre aplicación de medidas sanitarias y fitosanitarias (MSF) y el Acuerdo sobre obstáculos técnicos al comercio (OTC) emanados de la Ronda Uruguay, en los que se establecen medidas con efectos especialmente significativos en el comercio de productos alimentarios.

En ellos se reconoce el derecho de los miembros de la OMC para aplicar medidas de protección de la salud humana, animal o vegetal siempre que no sean arbitrarias y no discriminatorias esto significa que si un país aprueba restricciones a la importación de productos alimentarios por razón de que afecten a la salud humana, animal o vegetal sobre la base de motivos subjetivos o por presiones de grupos de interés puede ser denunciado por el país perjudicado por la restricción para que la medida sea retirada. El Acuerdo sobre obstáculos técnicos al comercio (OTC) tiene por objeto asegurar que las normativas nacionales no constituyan barreras o restricciones encubiertas al comercio internacional o un medio de discriminación.

\section{1- b) Normativa comunitaria}

La Unión Europea ha ido creando una amplia legislación en materia de seguridad alimentaria, legislación que ha ido evolucionando creando un cuerpo normativo del que pueden destacarse dos etapas:

- Directiva 93/43/CEE del Consejo aprobada en 1993 con la consolidación del Mercado Único Europeo y de la libre circulación de mercancías, capitales y servicios se hizo preciso establecer un sistema que asegurara la calidad de los productos alimentarios aprobándose esta Directiva relativa a la higiene de los alimentos que impuso la obligatoriedad del sistema ARCPC. La Directiva entiende por higiene de los productos alimentarios todas las medidas precisas para garantizar la seguridad y salubridad de los productos alimentarios que cubren todas las etapas de la cadena alimentaria. Dispone que las empresas de este sector están obligadas a indicar cualquier fase de su actividad que sea determinante para garantizar la seguridad de los alimentos velando asimismo para que se definan, se cumplan y actualicen los procedimientos de seguridad adecuados de acuerdo con una serie 
de principios que recoge y que están fundamentados en el sistema ARCPC. Además los Estados miembros deben fomentar la elaboración de guía de buenas prácticas sobre higiene que pueden ser tomadas como elementos de referencia por las empresas del sector agroalimentario como ayuda para garantizar el cumplimiento de las normas de seguridad alimentaria establecidas.

- El Libro Blanco de la Seguridad Alimentaria

La normativa comunitaria en materia alimentaria se ha ido aprobando para cubrir las necesidades coyunturales pero sin una planificación coherente, por ello cuando se quiso abordar esta cuestión de manera global se decidió la aprobación del Libro Blanco sobre Seguridad Alimentaria que aborda esta materia con un criterio de ordenación y coherencia y entre cuyos contenidos se incluyeron las siguientes propuestas:

- Creación de un organismo alimentario de ámbito europeo, encargado de las funciones de evaluación y comunicación mediante la emisión de dictámenes científicos y comunicados dirigidos a los consumidores. Con estos criterios, el Reglamento (CE) 178/2002 creó la Autoridad Europea de Seguridad Alimentaria y fijó los criterios principales relativos a los procedimientos seguidos en esta materia.

- Un nuevo marco jurídico que incluyó un plan de acción para mejorar y coordinar la normativa comunitaria aprobada en que la idea fuerza de la trazabilidad se incluyó en la expresión: de la granja a la mesa. El Libro Blanco presentó un anexo de más de 80 acciones dirigidas a completar y modernizar la legislación alimentaria comunitaria, disponiendo la necesidad de aprobar normativa sobre alimentación, salud y bienestar animal, higiene de los productos alimentarios contaminantes y residuos, así como aditivos, aromas, empaquetado, etc.

- El Libro Blanco previó asimismo una refundición de la normativa reguladora de control de la seguridad alimentaria a fin de garantizar que todos los eslabones de la cadena de producción de alimentos puedan ser objeto de control efectivo. Además también se estableció que la Comisión Europea debe promover la participación de los consumidores en el ámbito de la seguridad alimentaria. En estos aspectos se aprobaron la Directiva 89/107/CEE sobre aditivos autorizados en alimentos para consumo humano, la Directiva 94/35 sobre edulcorantes, la $94 / 36$ sobre otros aditivos alimentarios y la 89/397 sobre control oficial de productos alimenticios. 


\section{1- c) Normativa española}

La legislación sanitaria que impone obligaciones a la industria alimentaria es muy extensa y variada y afecta a muchos aspectos de su funcionamiento y entre otras pueden mencionarse las siguientes normas:

- Ley 11/2001 por la que se crea la Agencia Española de Seguridad Alimentaria con el objetivo de promover una gestión eficaz de la seguridad de los alimentos inspirada en los principios contenidos en el Libro Blanco comunitario.

- Real Decreto 709/2002 que aprueba el estatuto de la Agencia confiriéndole el carácter de Organismo Autónomo adscrito al Ministerio de Sanidad y Consumo. En el ámbito autonómico también se ha abordado esta materia por ejemplo la Ley 20/2002 de Cataluña donde se estableció la creación de la Agencia Catalana de Seguridad Alimentaria integrada en la Generalidad catalana.

- El Real Decreto 202/2000 sobre normas relativas a los manipuladores de alimentos: higiene de los manipuladores, responsabilidad de las empresas y modalidades de control del cumplimiento de esta normativa.

- El Real Decreto 1712/1991 regula el Registro General Sanitario de Alimentos.

- Real Decreto 2207/1995 sobre normas de higiene relativas a productos alimenticios, además de normas específicas referidas a sectores concretos: cárnicos, productos pesqueros, ovoproductos, lácteos, etc.

- Real Decreto 50/1993 regula el control oficial de productos alimenticios.

\section{PROYECTO DE ESTABLECIMIENTO DE UN SISTEMA DE GES- TIÓN DE LA CALIDAD}

Un sistema de gestión de calidad consta de dos partes una de ellas formada por el conjunto de textos escritos donde se recoge la documentación de resultados, datos y especificaciones establecidas en instrucciones de uso, planos, preceptos, normas, etc. Y otra que consiste en un esquema de las instalaciones, formación del personas, actividades, procesos y ejecución de casos concretos. Esta descripción proporciona las directrices más importantes de un proyecto pero no se ocupa de cuestiones como el control financiero ni de los aspectos sociales del sistema. 
Los puntos claves para llevar a cabo un proyecto de sistema de gestión de calidad son los siguientes:

1) Planificación del proyecto. En el caso de pequeñas empresas del sector agroalimentario no merece la pena crear grupos de trabajo ya que por lo general son organizaciones con recursos limitados y supone un gran costo económico y personal el añadido de tareas adicionales, por eso en estos casos es mejor contratar un consultor externo que realice las entrevistas y se encargue de someter a examen los resultados y de la redacción del informe final. Si bien este resultado es importante, aún lo es más el propio proceso con la participación del personal en las entrevistas intercambio de opiniones e ideas. La dirección de organización suele estar en manos de un Comité específico en el que conviene que tome parte un consultor externo que juegue el rol de miembro imparcial en la toma de decisiones de acuerdo con el orden del día establecido. Este comité debe encargarse de determinar los objetivos y describir el proyecto, de elaborar un plan y difundir información sobre el mismo en la organización, también debe elaborar la documentación sobre los requisitos de la normas ISO 9001:2000, crear grupos de trabajo evaluar las propuestas que se le presenten y llevar a cabo el seguimiento del proyecto del sistema de gestión de calidad que como orientación debe constar al menos de los siguientes apartados:

- Línea de procedimiento.

- Plan principal, mediante el uso del Diagrama Grantt (incluye todas las actividades principales y su responsable). Es el documento más importante y debe mantenerse actualizado.

- Presupuesto.

- Fases del proyecto.

- Seguimiento e información sobre el proyecto.

- Certificación.

2) Información a la organización de la empresa sobre el objetivo, alcance y resultados esperados del proyecto, sobre la serie de normas ISO 9000 y sobre las ventajas de la gestión de calidad, sobre el inicio de actividades y sobre los progresos del proyecto.

3) Comprobación del estado del sistema de gestión de calidad que tenga en ese momento la empresa. Análisis de la situación actual (elementos que existen, elementos que faltan, debilidades del sistema, informe sobre propuestas de mejora que debe incluir un borrador del plan principal del proyecto y observaciones sobre la concienciación del personal y estado de los equipos. 
4) Preparación de la documentación del sistema de gestión de calidad por parte de los diferentes grupos de trabajo, documentos relativos al desarrollo, las compras, el control de procesos, el servicio postventa en su caso, etc. Con frecuencia el director de calidad es nombrado coordinador de dichos grupos, que necesitan una instrucción específica que deberá incluir el campo de aplicación de la actividad, una interpretación de los requisitos especificados, los criterios de presentación, evaluación, modificación y aceptación de los resultados intermedios y definitivos y la estructura escogida para el sistema de gestión de calidad. Finalmente cuando se considere que los resultados están listos para ser presentados serán sometidos al comité que analizará si se han alcanzado los objetivos, si son precisas modificaciones y si el resultado es aplicable a las siguientes actividades.

5) Implantación práctica del sistema, que incluye un curso para los responsables de las actividades de implantación, instrucción para los usuarios de las actividades, introducción de equipos y herramientas y la realización de actividades demostrativas. En esta fase debe contarse con la presencia de un experto para resolver dudas y efectuar un seguimiento de las actividades. El comité deberá decidir el método a seguir de abajo arriba o de arriba abajo.

La implantación de procedimientos e instrucciones incluye la de instrucciones a los usuarios sobre el contenido, sobre el uso, el empleo de cualquier equipo o herramientas auxiliares, la realización de demostraciones prácticas y el auxilio de expertos. Además el grupo debe designar un responsable de seguimiento que garantice que el procedimiento implantado se utiliza correctamente que promueva la comprensión de los requisitos del procedimiento por parte de los usuarios, registrar errores u omisiones del proceso, describir las modificaciones necesarias e informar al grupo de trabajo.

6) Mantenimiento del sistema.

Durante el desarrollo de las auditorías internas de la calidad, especialmente las llevadas a cabo con anterioridad al proceso de certificación, los auditores deberán reunir las pruebas objetivas con objeto de aclarar si las modificaciones asociadas al mantenimiento se entienden, implantan y utilizan en la práctica por ello las listas de comprobación deberán estar específicamente concretadas.

El sistema de gestión de calidad debe ser auditado en su totalidad al menos una vez al año pero algunas partes más problemáticas lo serán con más frecuencia. El director de calidad será el responsable de elaborar y ejecutar el plan de auditorías aceptable que incluya medios para registrar sus logros. Además deben elaborarse planes espe- 
cíficos de auditoria para elementos concretos del sistema asegurando que la documentación se encuentra disponible en los lugares de uso, las actividades se llevan a cabo de conformidad con tales textos y que son adecuadas y eficaces para la implantación de una política de calidad y dando prioridad a las actividades de importancia especial para lograr el nivel de calidad especificada.

Los grupos de trabajo deben prepararse para la auditoria y esta debe llevarse a cabo de conformidad con el procedimiento ateniéndose a las correspondientes listas de comprobación.

Concluida la auditoria el grupo auditor realizará un informe que defina el alcance de aquella, demuestre el grado de conformidad existente entre el sistema de gestión de la calidad, la aplicación del sistema, los requisitos, el grado de implantación, establezca formularios de corrección y finalmente incluya conclusiones y recomendaciones. Este informe debe ser presentado a la dirección de la empresa para la consideración de posibles acciones correctoras en la organización, el sistema de gestión de la calidad, la asignación de recursos y la formación del personal.

7) Resúmenes y conclusión

La implantación de un sistema de gestión de la calidad incluye aspectos tales como la planificación, información, evaluación, descripción, implantación y mantenimiento.

Muchos empresarios se quejan de que una vez llevado a efecto un proceso de certificación los resultados esperados no se materializan (reducción de costes en un porcentaje establecido, etc. Las razones podrían hallarse en que la dirección no acepta incondicionalmente el proceso de normas ISO 9000 y ni enseña el camino ni asigna los recursos necesarios para la implantación en la empresa de los nuevos procesos requeridos y de la documentación necesaria.

Para elaborar el sistema de gestión de la calidad hay sistemas preestablecidos en soportes informáticos que pueden resultar herramientas útiles pero no garantizan su adaptación al perfil de la empresa individual. No obstante pueden resultar recomendables los sistemas que se limitan al control de la forma y estructura del sistema dejando la especificación del contenido en manos de expertos en los procesos. De esta manera es posible conseguir un sistema flexible, sencillo que puede actualizarse con rapidez y facilidad.

Finalmente hay que hacer referencia a la existencia de controles que la Administración Pública encomienda a entes específicos, que se comprometen a garantizar determinadas características de los productos por ellos certificados. Por lo general se trata de organi- 
zaciones que agrupan a diversos profesionales y que encargan el controlo a un órgano creado al efecto, En este grupo se encuentran las Denominaciones de origen, las Denominaciones específicas, las Marcas de calidad e incluso, antes de carácter generalmente público, y a veces también privado, que garantizan determinadas características de los alimentos. La certificación que amparan debe ser cumplida en todos los parámetros y responden de su cumplimiento ante la Administración pública correspondiente. En todo caso deben garantizar niveles de calidad superiores a los exigidos por la legislación o incluso características alimentarias que aunque no se hallen legisladas, supongan una mejora objetiva considerable del producto.

\section{3) NORMAS ISO RELACIONADAS CON LA CALIDAD DE PRO- DUCTOS Y SERVICIOS}

- $\quad$ ISO 9000:2000 Sistemas de gestión de la calidad. Fundamentos y vocabulario (UNE-EN ISO 9000:2000).

- ISO 9000:2000 Gestión de la calidad y aseguramiento de la calidad. Parte 3: Directrices para la aplicación de la Norma ISO 9001:1994 al desarrollo, suministro, instalación y mantenimiento de soporte lógico (UNE-EN ISO 9000-3:1998).

- ISO 9000-4:1993 Normas para la gestión de la calidad y aseguramiento de la calidad. Parte 4: Guía para la gestión de seguridad de funcionamiento.

- ISO 9001:2000 Sistemas de gestión de la calidad. Requisitos (UNEEN ISO 9000-1:2000).

- $\quad$ ISO 9004:2000 Sistemas de gestión de la calidad. Directrices para la mejora del desempeño (UNE-EN 9004:2000).

- ISO 10005:1995 Gestión de la calidad. Directrices para los planes de la calidad (UNE 66904-5:1996).

- ISO 10006:1997 Gestión de la calidad. Directrices para la calidad en la gestión de proyectos (UNE 66904-6:2000).

- SO 10007:1995 Gestión de la calidad. Directrices para la gestión de la configuración (UNE-EN ISO 10007:1997).

- $\quad$ ISO 10011-1:1990, 10011-2:1991, 10011-3:1991 Reglas generales para la auditoria de los sistemas de la calidad. Partes 1,2 y 3: Auditoria revisada como norma ISO 19011 (EN 30011-1, 2 y 3:1993).

- $\quad$ ISO 10012-1:1992, ISO 10012-2:1997 Requisitos de aseguramiento de la calidad para los equipos de medida Parte 1 Sistema de confirma- 
ción metrológica de los equipos de medida, parte 2 Directrices para el control de los procesos de medida.

- $\quad$ ISO 10013:1995 Directrices para el desarrollo de los manuales de calidad.

- ISO/TR 10014:1998 Directrices para la gestión de la economía de calidad.

- $\quad$ ISO 10015:1999 Gestión de la calidad. Directrices para la formación.

- ISO/TR 10017:1999 Directrices sobre técnicas estadísticas para la norma ISO 9001:1994.

- ISO 14001:1996 Sistemas de gestión medioambiental. Especificaciones y directrices para su utilización (UNE-EN ISO 14001:1996).

- ISO 19011:2002 Directrices para la auditoria de sistemas de gestión de la calidad y/o medioambiental.

- CEI 60300-1 Gestión de la seguridad de funcionamiento.

- ISO/CEI 17025:1999 Requisitos generales para la competencia de laboratorios de comprobación y calibración.

- ISO/TS 16949:1999 Automoción (anterior QS 9000) Requisitos de los sistemas de la calidad.

- EN 729-1, 2 , 3 y 4:1994 Requisitos de calidad para el soldeo: soldeo por fusión de materiales metálicos parte 1 Directrices para su selección y utilización, parte 2 requisitos de calidad completos, parte 3 requisitos de calidad standard, parte 4 requisitos de calidad elementales (UNE-EN- 1 , 2, 3 y 4:1995).

- EN 45002:1989 Criterios generales para la evaluación de los laboratorios de ensayo (UNE 66502:1991).

- EN 45003:1995 Sistemas de acreditación de laboratorios de ensayo y calibración. Requisitos generales relativos a su funcionamiento y reconocimiento (UNE-EN 45003:1995).

- EN 45004:1995 Criterios generales para funcionamiento de los diversos tipos de organismos que realizan inspección (UNE-EN 45004:1995).

- EN 45010:1998 Requisitos generales para la evaluación y acreditación de entidades de certificación (UNE-EN 45010:1998).

- EN 45011:1998 Requisitos generales para las entidades que realizan la certificación de producto (UNE-EN 45011:1998).

- EN 45012:1998 requisitos generales para entidades que realizan la evaluación y certificación de sistemas de calidad (UNE-EN 45012:1998).

- EN 45013:1989 Criterios generales relativos a organismos de certificación que realizan certificación de personal (UN E 66513:1991). 
- EN 45014:1998 Criterios generales para efectuar la declaración de conformidad del suministrador (UNE-EN 45014:1998).

- EN 45020:1997 Normalización y actividades relacionadas. Vocabulario general (UNE-EN 45020:1998).

- $\quad$ EN 46001:19996 y EN 46002:1993 Sistemas de calidad: productos sanitarios requisitos particulares de aplicación de las normas EN ISO 9001 y 9002 . 